\title{
Associative and postprandial control of schedule-induced drinking: Implications for the study of interim behavior
}

\author{
THOMAS R. MINOR and XENIA COULTER \\ State University.of New York, Stony Brook, New York
}

\begin{abstract}
Schedule-induced drinking and food-magazine contacts were examined in rats receiving either true- or pseudodiscriminative conditioning. Schedule-induced drinking was largely confined to S- following true discrimination training, but when S1 and S2 were unrelated to food deliveries (pseudo condition), drinking occurred after food ingestion. A stimulus generalization test for drinking yielded an excitatory postdiscrimination gradient around $\mathrm{S}-$ after the true discrimina. tion and a flat gradient after the pseudo discrimination. Additional observations showed Sdrinking to be closely related to the amount of food consumed during the immediately preceding S+ trial. These data suggested that both the predictive feature of $\mathbf{S}-$ and postprandial stimuli can control schedule-induced drinking. It was argued that these variables represent two general processes-induction and selection-both of which are necessary conditions requiring further study. Magazine contacts during S+ did not distinguish the true from the pseudo condition and were not influenced by test stimuli during a generalization test.
\end{abstract}

When hungry animals are exposed to a periodic food schedule, two highly stereotyped behavioral patterns develop during the intervals between food presentations (see Staddon, 1977, for review). Terminal, or foodoriented, responses appear in the final seconds before each food delivery. Often these responses resemble behavior observed during eating: pigeons peck at the feeder wall at such times (e.g., Staddon \& Simmelhag, 1971) and rats scrabble and gnaw at the food magazine (e.g., Reberg, Mann, \& Innis, 1977; Staddon \& Ayres, 1975). Interim activities immediately follow food ingestion. Interim activities vary to some extent with the species, but seem to be determined mainly by the presence of some appropriate goal object. For example, excessive drinking (i.e., schedule-induced drinking) is one activity that readily develops in several species if a water bottle is located near the feeder area (Falk, 1971). Other interim behaviors that have been reported include wheel running in rats (Levitsky \& Collier, 1968), agitated behavior in pigeons (Killeen, 1975; Staddon \& Simmelhag, 1971), and aggressive behavior towards a conspecific or inanimate object (e.g., Cohen \& Looney, 1973; Flory, 1969; Hutchinson, Azrin, \& Hunt, 1968).

This manuscript was prepared while T. R. Minor was a Killam postdoctoral fellow at Dalhousie University. The research was supported by NIMH Grant 5 RO1 MH2950403 to X. Coulter and was a portion of a doctoral dissertation submitted to the Graduate School at the State University of New York at Stony Brook in partial fulfillment of the requirements for the $\mathrm{PhD}$ degree. The authors wish to thank H. C. Rachlin, V. M. LoLordo, and J. W. Jacobs for their helpful comments. Reprint requests should be addressed to T. R. Minor, Department of Psychology, Campus Box 345, University of Colorado, Boulder, Colorado 80309.
The present study was concerned with the conditions underlying the organization and control of these interim and terminal behaviors. Staddon (1977) has argued that different response classes are engendered by different expectations about the temporal proximity of a reinforcer; terminal responses occur when the animal expects imminent food, whereas interim responses occur in anticipation of the momentary absence of food. According to this interpretation, a stereotyped behavioral pattern develops within the interfood interval because time since the last food delivery acts as a cue for the next food delivery. More specifically, when food is made available at fixed time intervals, each food presentation begins a brief period during which a subsequent food presentation is highly unlikely (an $\mathrm{S}$ - period). Presumably this negative stimulus-reinforcer relation engenders a specific motivational state (viz. an interim state) in which activities such as excessive drinking, wheel running, and aggression are likely to occur. Later in the interval, the temporal cues predict forthcoming food (an S+ period). This positive stimulus-reinforcer relation induces a second, terminal state in which food-related behaviors are likely to occur. Finally, since terminal and interim behaviors occur at different times, Staddon has also argued that these activities, or more properly their corresponding states, are antagonistic and compete for available session time.

Staddon's suggestions concerning terminal responding during periodic schedules are supported by studies using more conventional Pavlovian procedures, particularly investigations of sign-tracking and autoshaping (see Schwartz \& Gamzu, 1977, for a review). Overall, this literature indicates that several aspects of consummatory behavior are readily brought under associative control by 
stimuli predicting food. Such studies usually employ a clearly localized stimulus as an $\mathrm{S}+$, which results in consummatory behavior being directed at the stimulus (e.g., Brown \& Jenkins, 1968). Of course, no such stimulus is available in the simple periodic schedule typically used to study schedule-induced behavior. However, postfood time could serve a similar function. If so, consummatory behavior would be induced during the time periods just preceding the delivery of food. Because of the nonlocalized nature of temporal cues, these behaviors might lack the directionality of autoshaped responses or might be directed at the reinforcement site (as in goal tracking; see Boakes, 1978). From this perspective, there seem to be solid grounds for assuming that terminal behavior is induced by the prediction of a forthcoming food presen. tation.

Support for Staddon's proposals concerning interim behavior is less direct. It is based mainly on two observations: (1) interim activities occur at times when the food is unlikely to be delivered, and (2) the motivation for interim behavior increases with the motivation for terminal behavior (cf. Falk, 1971; Staddon, 1977). This second observation is consistent with several current models of Pavlovian conditioning. That is, the level of inhibitory conditioning resulting from a negative stimulus-reinforcer relation (control by $\mathrm{S}_{-}$) is often assumed to be a direct function of the level of excitatory conditioning, resulting from a positive stimulus-reinforcer relation (control by S+) (Rescorla \& Wagner, 1972). Thus, the amounts of terminal and interim behavior should vary together. It should be noted, however, that neither observation forces the conclusion that interim activities are controlled by stimuli predicting nonreinforcement. It is also plausible, for instance, that terminal behavior is inhibited by the delivery of food. The resulting behavioral void is likely to be filled by some other form of behavior. As long as drinking, wheel running, etc., are preferred to doing nothing, such activities might be expected to occur when the principal reinforcer is predictably unavailable. From this perspective, interim activities are more properly regarded as vacuum activities (e.g., Tinbergen, 1952) rather than behaviors induced and controlled by temporal S- stimuli.

The main objective of the present study was to determine whether interim responses were, in fact, controlled by signals for nonreinforcement. Unfortunately, such a determination is not easily made when behavior is maintained by simple food schedules, since time is the variable predicting food and its absence. Given a Pavlovian interpretation, however, it should be possible to examine interim behavior in a paradigm in which the absence of food is predicted by an explicit stimulus, much as terminal behavior has been studied in autoshaping procedures. Once a behavior is clearly localized in the presence of an explicit stimulus, there are several procedures available for assessing stimulus control. The present study employed a two-component multiple schedule in which food was presented at irregular intervals in one stimulus $(\mathrm{S}+)$, but not the other $(\mathrm{S}-)$. This procedure has been used extensively to study terminal behavior (e.g., Gamzu \& Schwartz, 1973) and closely approximates the reinforcement conditions in a simple periodic schedule. Schedule-induced drinking by rats was used as a measure of interim responding, and contacts with the food magazine indexed terminal responding. Following discrimination training, a stimulus generalization test was used to assess the relationships among $\mathrm{S}+, \mathrm{S}-$, and these behaviors. According to Staddon's (1977) Pavlovian interpretation, St should accrue excitatory control over mag. azine contacts and inhibitory control over scheduleinduced drinking. Symmetrically, S- should accrue excitatory control over schedule-induced drinking and inhibitory control over magazine contacts. Alternatively, if interim activities occur as vacuum activities, there is little reason to expect that $\mathrm{S}$ - should exert associative control over these activities at all.

As a control for the nonassociative effects of presenting novel stimuli during the generalization test, other rats were given a pseudo discrimination. This differed from the true discrimination only in that $\mathrm{S} 1$ and $\mathrm{S} 2$ bore no consistent relationship to the presence or absence of food. This procedure maintained the essential features of a truly random control (Rescorla, 1967), and therefore provided a sensitive baseline from which the excitatory or inhibitory effects of a stimulus on a particular response class could be measured.

\section{METHOD}

\section{Subjects}

Twenty-four experimentally naive adult male Long-Evans rats from the breeding colony of the Psychology Department, SUNY at Stony Brook, were reduced to and maintained at $80 \%$ of their free-feeding body weights. Between sessions, the subjects were housed in individual cages where water was freely available.

\section{Apparatus}

The apparatus consisted of four standard rat chambers housed in light- and sound-attentiating chests. Exhaust fans on the chests provided masking noise at approximately $75 \mathrm{~dB}$ SPL. A recessed food magazine was located on the front wall of each chamber, $7 \mathrm{~cm}$ to the left of center and $2 \mathrm{~cm}$ above the level of the grid floor. Standard 45-mg Noyes food pellets could be delivered into the magazine from a solenoid-operated feeder located outside each chamber. A ball-tipped water tube, $6 \mathrm{~mm}$ in diameter, was inserted into the chamber through a $1.2-\mathrm{cm}$ aperture located $7 \mathrm{~cm}$ to the right of center on the front wall and $8 \mathrm{~cm}$ above floor level. The water tubes were attached to a calibrated reservoir outside each chamber. Contacts with the food magazine and water tube were monitored by BRS-LVE (101) drinkometers. A 6-W lamp covered by a white plastic dome served as a houselight and provided constant dim illumination throughout each session. The houselight was located in the center of the front wall, $14 \mathrm{~cm}$ above floor level.

Light and tone served as the component stimuli of the multiple schedule. The light stimulus consisted of the operation of two 6-W lamps located on either side of the clear Plexiglas ceiling of each chamber. A $1,500-\mathrm{Hz}$ tone was generated by a solid state programmable tone generator, amplified by a GrasonStadler 1-W amplifier, and delivered through a speaker located in 
the center of the ceiling of each chamber. During the generalization test, six additional tones, equally spaced on a log scale (i.e., $611,825,1,112,2,023,2,730$, and $3,682 \mathrm{~Hz}$ ), were presented in a similar fashion. All tones were presented at an intensity of approximately $80 \mathrm{~dB}$ SPL re $.0002 \mathrm{dynes} / \mathrm{cm}^{2}$. Experimental events were programmed with solid-state logic located in an adjoining room.

\section{Procedure}

Rats were assigned randomly to one of four groups of six rats each. Two groups were exposed to the true discrimination (TD). For one of these groups (TDT+), tone served as S+, and light as $\mathrm{S}-$; for the other group (TDT-), tone was $\mathrm{S}_{-}$, and light, S+. The other two groups were exposed to a pseudodiscrimination (PD) in which light and tone were unrelated to food or its absence. One of these control groups (PDT+) was arbitrarily treated during the generalization test as if it had received TDT+ training; the remaining control group (PDT -) was treated as if it had received TDT - training. All groups were exposed to the following conditions.

Baseline. The subjects were initially given three daily 1 -h sessions to explore and habituate to the experimental chambers. Water and 60 food pellets were freely available in each session. The food pellets were placed in the magazine before the subject entered the chamber. Water-tube contacts (licks) and magazine contacts were recorded as indicants of the preexperimental (baseline) response levels.

Discrimination training. On Day 4 all rats began 20 daily sessions of discrimination training. Rats receiving TD training were exposed to a multiple extinction variable-time 30-sec (mult EXT VT 30-sec) schedule of food delivery. During the VT 30-sec schedule, which was generated by sampling a probability gate set at $20 \%$ every $6 \mathrm{sec}$, food deliveries were independent of the subjects' behavior. Groups PDT+ and PDT- were exposed to a similar food schedule; however, neither light nor tone was consistently related to EXT and VT periods. The first three sessions of discrimination training consisted of 103 -min presentations of light and $103-\mathrm{min}$ presentations of tone in strict alternation. These initial long intervals were used to facilitate discrimination between components. The remaining sessions consisted of 30 1-min light presentations and $301-\mathrm{min}$ tone presentations in strict alternation. Water was freely available in the chambers for all rats during both discrimination training and the subsequent generalization test. Total number of magazine and lick responses per session were recorded separately in EXT and VT. These data were occasionally supplemented with cumulative records of licking and informal observations of the animals.

Generalization test. The generalization test was administered during the seven sessions immediately following discrimination training. With the exception of test trials, the conditions for each group were the same as in discrimination training. In each session, seven test trials occurred, such that the training tone and each of the six test tones were presented once per session; the order of these stimulus presentations was counterbalanced across sessions for each subject. When a test stimulus was presented, it replaced a semirandomly selected S+ (or VT) trial for Groups TDT+ and PDT+, and a semirandomly selected S- (or EXT) trial for Groups TDT - and PDT -. The constraints on random selection were that no test stimuli could occur during the first $5 \mathrm{~min}$ of a session or sooner than $3 \mathrm{~min}$ after the preceding test trial. Food presentations were discontinued during test trials for Groups TDT+ and PDT+.

One rat (TD62) was given three additional discrimination sessions after the generalization test for reasons that will become apparent later.

\section{RESULTS}

\section{Baseline}

Single-factor analyses of variance ${ }^{1}$ revealed no reliable differences among groups in either magazine con- tacts or licks during the last baseline session. Mean number of magazine contacts in this session for Groups TDT+, TDT-, PDT+, and PDT - were, respectively, 511.2, $213.7,352.0$, and 410.7 ; mean number of licks were, respectively, 551.5, 228.3, 504.0, and 389.7.

\section{Discrimination Training}

There were no obvious differences between Groups TDT+ and TDT - or between Groups PDT+ and PDTin either magazine contacts or drinking during discrimination training. Consequently, the data from this phase were considered only in terms of TD vs. PD.

Figure 1 presents mean discrimination ratios for magazine and lick responses in the TD and PD conditions for each session of discrimination training. In both the TD and PD conditions, food (VT) and no food (EXT) periods occurred in exactly the same manner; hence, the discrimination ratios were defined in terms of these periods. The ratio (VT - EXT)/(VT + EXT), the number of responses in VT minus the number of responses in EXT divided by the combined total, reflects the relative distribution of responses between components of the multiple schedule. The ratio can vary between +1 , which indicates that all instances of a particular behavior occurred in VT, and -1 , which indicates all responses occurred in EXT. A ratio of 0 represents an equal distribution of responses between EXT and VT.

Most rats failed to show a reliable increase in magazine contacts from the baseline levels. However, by the last discrimination session, mean total magazine contacts in the TD and PD conditions were, respectively, 1,185.08 and 382.17 . This difference was statistically reliable

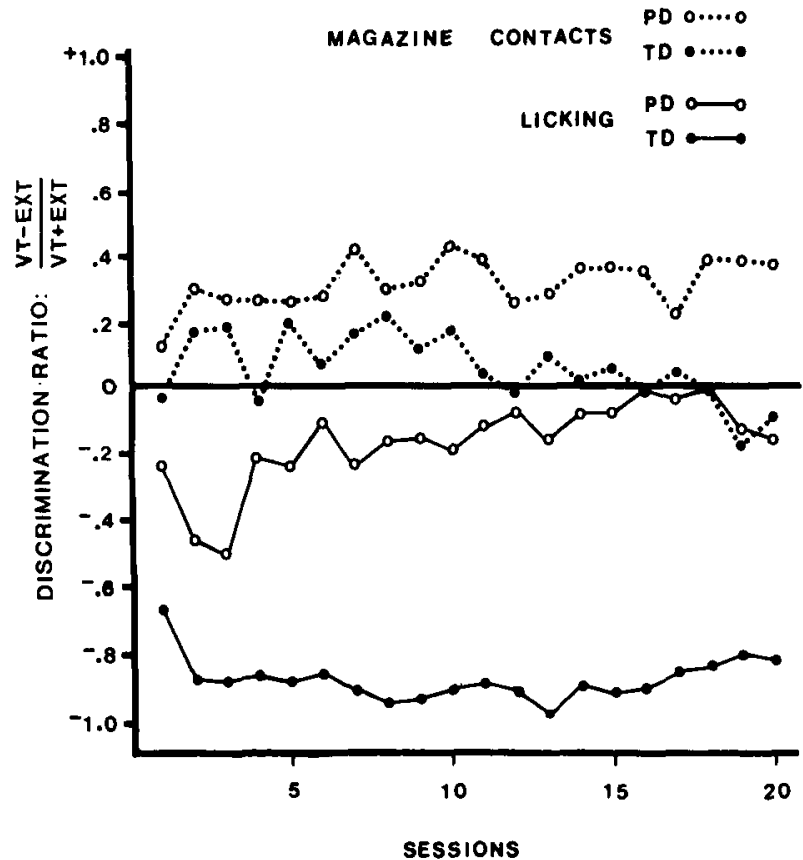

Figure 1. Mean discrimination ratios for magazine and lick responses for each session of training in the true (TD) and pseudo (PD) discrimination. 
$[t(22)=2.31, p<.05]$. The higher mean in the TD condition was largely due to four rats that did show a large increase from baseline, but made the majority of these responses in S- rather than S+. This pattern is reflected in the discrimination scores shown in Figure 1. Magazine contacts did not predominate in VT, as had been predicted for TD rats; in fact, discrimination scores were higher in the control condition. Thus, magazine contacts turned out to be a poor measure of terminal behavior.

Observation of the rats revealed certain differences in the terminal behavior of discrimination and control subjects. With TD training, rats displayed a strong tendency to crouch a few centimeters in front of the food magazine in St. Food presentations resulted in a brief contact with the magazine followed by a return to this crouch position. No such crouching was evident in S-. However, the four rats in the TD condition showing an increase from baseline in magazine contacts spent the latter portion of each $\mathbf{S}-$ trial digging and pawing at the magazine. With PD training, rats displayed no consistent orientation towards the food magazine, spending much of their time in both components moving back and forth between the water tube and magazine. Thus, the failure to observe a reliable difference in terminal behavior between conditions, as indicated by magazine contacts, appeared to be a measurement problem rather than a failure of S+ to engender a reliable pattern of foodoriented behavior.

Drinking increased from baseline for all rats such that there was no overlap between the baseline levels and those in the last discrimination session. Differences in total session drinking during discrimination training were evident between conditions. In the last discrimination session, the mean combined lick totals (i.e., EXT+VT) in TD and PD were, respectively, 2,542.33 and 4,128.33. The greater drinking in PD was statistically reliable $[\mathrm{t}(22)=2.49, \mathrm{p}<.05]$. Moreover, as shown in Figure 1 , rats given TD training drank primarily in $S-$. Rats given PD training, on the other hand, drank approximately equal amounts in EXT and VT. Figure 2 shows the cumulative records of licking for a typical rat from each training condition. The upper pen indicates licking and was reset at the end of each component. Food presentations are shown as downward slashes of the upper pen. The schedule conditions are shown by the lower event pen. For Rat PD11 drinking was organized mainly by food presentations in VT. The considerable drinking seen in EXT appeared to be simply a continuation of a drink bout initiated by a food presentation in the preceding VT period. In contrast, drinking by Rat TD22 was clearly organized by the discriminative stimuli; that is, drinking was initiated by the onset of S- and usually terminated at or before the end of the component. The occasional drinking seen in S+ was mainly a brief spillover from the preceding $S-$ trial and usually terminated with the first food delivery in St.

The response patterns in Figure 2 suggested that the differences between TD and PD conditions in total session drinking may have resulted from differences in the number of eliciting stimulus presentations. That is, rats showing a postpellet drinking pattern had, on average, 60 such presentations, since approximately that many food deliveries occurred in each session. In contrast, rats drinking with the onset of $S-$ had only 30 presentations.
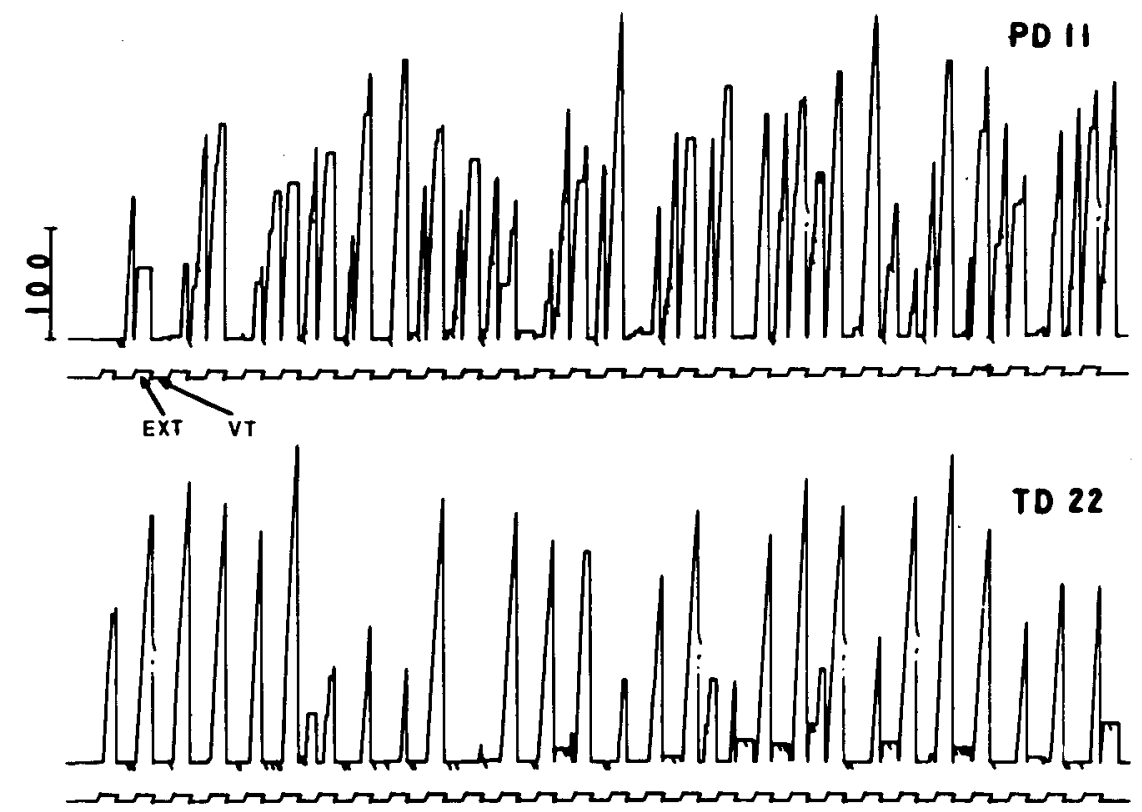

Figure 2. Cumulative records of licking for a typical rat from the TD and PD conditions. Drinking followed food deliveries for Rat PD11 (top panel), but followed the onset of $\mathrm{S}-$ for Rat TD22 (bottom panel). 
Thus, even though these different events may have produced equivalent drink bouts, differences in total session drinking would have emerged simply because there were more food presentations than S- presentations per session. To determine whether the difference in total session drinking was simply the result of this inequity, or also stemmed from differences in bout consumption in the two conditions, lick totals were converted to licks/Sfor TD rats and to licks/pellet for PD rats. This yielded a rough estimate of average bout consumption. Using the data from the last discrimination session, these conversions produced slightly higher response rates in TD (means for TD and PD, respectively, were 85.25 and 66.69). However, this difference was not statistically reliable $[t(22)=.42, p>.05]$. Thus, an $S-$ following TD training and food presentations following PD training appeared to be equally effective in eliciting drinking.

\section{Generalization Test}

The median number of magazine and lick responses for the seven sessions of generalization testing was computed at each tone frequency value for each rat. The group means and standard deviations for these data are presented in Table 1. The postdiscrimination gradients for magazine contacts are shown in the upper portion of the table. As is evident, variation in tone frequency during the generalization test had no consistent effect on magazine contacts in any of the four groups, as was confirmed by a groups $X$ stimulus $X$ tone frequency repeated-measures analysis of variance (all Fs $<2.00$, ps $>.05$ ). Thus, as suggested by the discrimination scores reported earlier, the generalization test confirmed that tone failed to accrue either excitatory control over magazine contacts when it predicted food (Group TDT + ) or inhibitory control when it predicted the absence of food (Group TDT-).

The postdiscrimination gradients for interim drinking are shown in the lower portion of Table 1. Changing tone frequency had no effect on drinking for rats tested during S+ (or VT) trials. Virtually no drinking occurred at any of the tone values tested for Group TDT + , and very little drinking occurred in the control group, PDT + . A group $X$ tone frequency mixed-design analysis of variance revealed a main effect of group $[F(1,10)=$ 8.24], but showed neither a main effect of tone frequency nor an interaction between the two variables. Although this analysis indicates that Group PDT+drank more than Group TDT+ during the generalization test, the drinking levels for PDT+ animals were considerably lower than during discrimination training. However, it should be recalled that these rats were tested during nonreinforced VT trials. Since food deliveries initiated drinking for these animals, the relative lack of drinking during the generalization test can be attributed to the absence of these eliciting events. The absence of drinking in Group TDT+ may reflect either inhibitory control of interim behavior by $\mathrm{S}+$, which generalized to all the tone values tested, or the simple lack of any sort of control by $S+$ over interim behavior. Unfortunately, the present procedure does not distinguish between these alternatives. Of greater interest is the finding for the rats tested during S- (or EXT) trials. When tone predicted the absence of food, tone frequency had a substantial effect on drinking for Group TDT-, but had little effect in the control condition (PDT-). Generally, smaller amounts of drinking occurred at the tone values furthest from $\mathrm{S}-$ in Group TDT-, resulting in a peaked decremental gradient with a maximum at $\mathbf{S}-$. Such a function has traditionally served as the operational definition of excitatory stimulus control (e.g., Terrace, 1966). In contrast, the gradient for Group PDT - was essentially flat, indicating a lack of stimulus control. These trends were confirmed by a group $X$ tone frequency mixed-design analysis of variance which yielded significant main effects of group $[F(1,10)=8.47]$ and tone frequency $[F(6,60)=2.71]$, and, more importantly, a significant group $X$ tone frequency interaction $[F(6,60)=2.53]$. Post hoc contrasts (Rodger, 1975) performed on the interim gradients for Group TDTindicated a reliable effect of tone frequency on drinking.

Table 1

Postdiscrimination Gradients for Terminal and Interim Behavior

\begin{tabular}{|c|c|c|c|c|c|c|c|c|c|c|c|c|c|c|}
\hline \multirow[b]{3}{*}{ Group } & \multicolumn{14}{|c|}{ Tone Frequency } \\
\hline & \multicolumn{2}{|c|}{611} & \multicolumn{2}{|c|}{825} & \multicolumn{2}{|c|}{1112} & \multicolumn{2}{|c|}{1500} & \multicolumn{2}{|c|}{2023} & \multicolumn{2}{|c|}{2730} & \multicolumn{2}{|c|}{3682} \\
\hline & Mean & SD & Mean & SD & Mean & SD & Mean & SD & Mean & SD & Mean & SD & Mean & SD \\
\hline & \multicolumn{14}{|c|}{ Magazine Contacts } \\
\hline TDT+ & 4.33 & 6.95 & 5.33 & 7.73 & 2.67 & 5.54 & 4.17 & 6.42 & 3.67 & 4.23 & 4.67 & 8.57 & 2.17 & 4.23 \\
\hline PDT+ & 2.17 & 2.43 & .83 & .76 & 6.67 & 13. & 2.67 & 3. & & 1. & 2.83 & 2. & .83 & 1.33 \\
\hline TDT - & 27.00 & 44.00 & 19.33 & 38.20 & 27.00 & 52.95 & 26.17 & 45.12 & 19.00 & 31.92 & 27.17 & 42.05 & 19.00 & 24.26 \\
\hline \multirow[t]{2}{*}{ PDT- } & 1.83 & 3.46 & 5.67 & 12.29 & 2.00 & 1.90 & 5.00 & 10.32 & 3.83 & 6.62 & 4.00 & 8.42 & 3.00 & 6.87 \\
\hline & \multicolumn{14}{|c|}{ Licks } \\
\hline TDT + & .00 & .00 & .00 & .00 & .17 & .41 & .00 & .00 & .00 & .00 & .00 & .00 & .00 & .00 \\
\hline PDT+ & 2.50 & 4.68 & 1.17 & 1.83 & 10.17 & 21.07 & 1.00 & .90 & 1.50 & 3.21 & 12.17 & 17.76 & 2.50 & 4.18 \\
\hline TDT- & 43.33 & 38.75 & 92.17 & 36.92 & 87.50 & 39.85 & 149.33 & 48.35 & 63.33 & 56.66 & 70.67 & 99.27 & 20.67 & 19.20 \\
\hline PDT_- & 25.17 & 38.94 & 43.67 & 66.95 & 26.50 & 26.99 & 32.50 & 33.59 & 20.17 & 14.53 & 39.33 & 59.40 & 37.67 & 34.80 \\
\hline
\end{tabular}




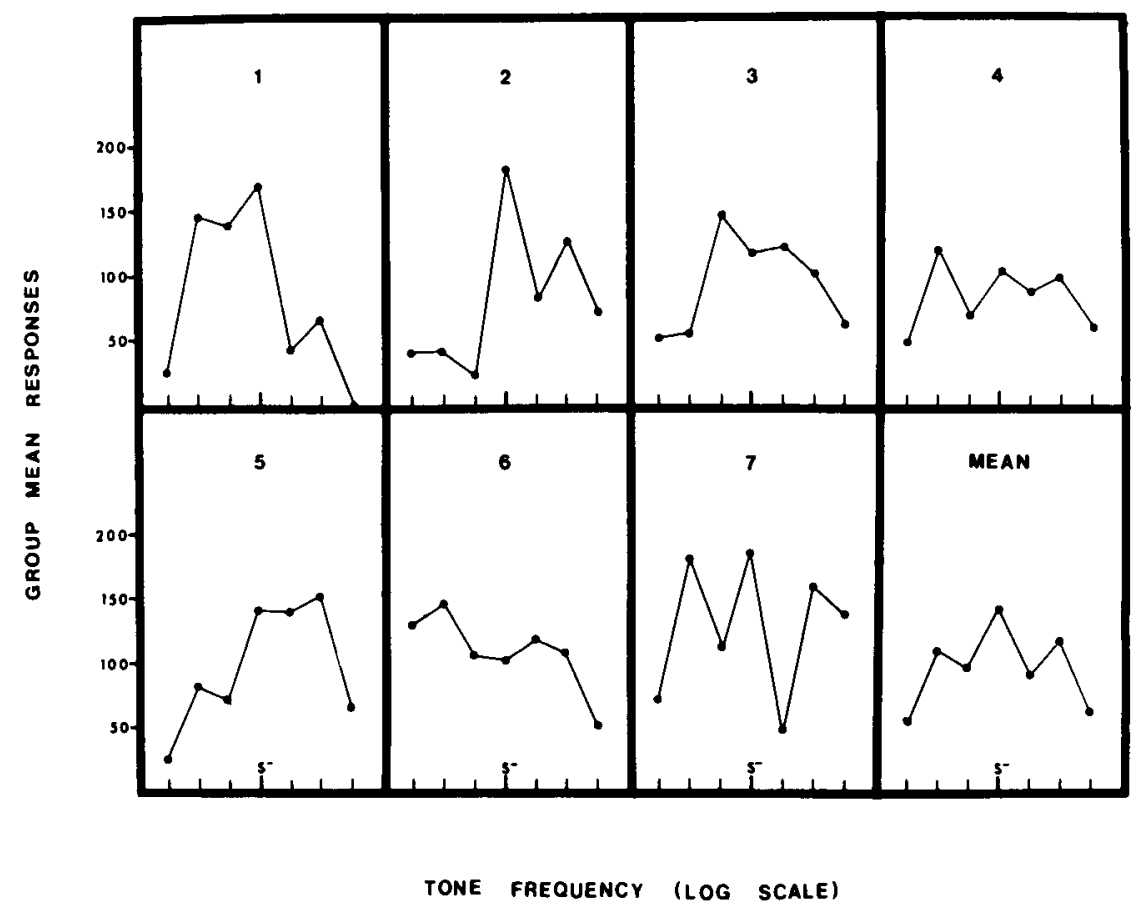

Figure 3. Mean interim gradients of drinking for Group TDT - for each of seven sessions of generalization testing.

This analysis implied the following order of population drinking means at the various tone frequencies: $1,500>$ $1,112=2,023>825=2,730>611=3,682$.

Figure 3 shows the mean interim gradients for Group TDT- for each of the seven sessions of generalization testing. There was a noticeable flattening of the gradients over consecutive sessions due to an increase in drinking to the test stimuli relative to $S-$. These increases appeared to occur differentially such that drinking increased more rapidly to the tone values closest to $S-$. This produced a flattening in the middle of the gradients prior to the extremes (e.g., Sessions 3, 4, and 5).

Tone frequency did not appear to affect the local rate of licking, which was estimated from cumulative records at $4-7$ licks/sec. Once drinking was initiated during a test trial, it continued unabated. Tone frequency did appear to change the latency to the first lick during test trials; that is, frequencies distant from $\mathrm{S}-$ tended to displace drinking from the onset of the stimulus to later in the trial. Although latency data were not directly available, it was possible to simply count the number of trials during which no drinking occurred (i.e., trials during which drinking occurred with a maximum latency of $60 \mathrm{sec}$ ). These data produced an incremental gradient with a minimum at $S-$ : there were $15,9,12,3,17,17$, and 20 trials with no licks for the tone frequencies ranked lowest to highest.

Finally, there was some evidence that the number of food pellets presented during the S+ trial which preceded a test trial was an important determinant of drinking during that trial. Specifically, it appeared that the more food pellets delivered, the shorter was the latency to drink regardless of tone frequency. This finding also suggested that the number of pellets delivered in S+ may have been an important determinant of drinking in Sduring discrimination training. To assess this possibility, Rat TD62 was given three additional discrimination sessions after the generalization test. The number of food pellets presented on each $S+$ trial and the number of licks occurring in the subsequent $\mathrm{S}$ - trial were recorded. The resulting data are presented in Table 2 . It can be seen that drinking in S- increased directly with the number of food pellets in St. Pearson product-moment correlation coefficients were computed for each session

Table 2

Mean Licks in S- as a Function of the Number of Pellets in S+

\begin{tabular}{lccrrrr}
\hline & \multicolumn{4}{c}{ Number of Pellets in S+ } \\
\cline { 2 - 6 } & \multicolumn{1}{c}{0} & 1 & 2 & 3 & 4 \\
\hline Frequency Over Three Sessions & 13 & 25 & 17 & 19 & 6 \\
Mean Licks & 25.15 & 74.28 & 117.59 & 205.26 & 216.00 \\
Standard Deviation & 31.20 & 73.03 & 98.20 & 93.48 & 70.08 \\
\hline
\end{tabular}


and confirmed a fairly strong relationship between eating and subsequent drinking: that is, $.6850, .6215$, and .5266 for Days 1,2 , and 3, respectively.

\section{DISCUSSION}

These data show that schedule-induced drinking, an interim behavior, is organized and controlled by stimuli predicting the absence of food reinforcement. Interim drinking developed in and was confined to $S-$ for rats learning a discrimination for the presence and absence of food. The peaked form of the postdiscrimination gradient for Group TDT-provides direct evidence that such drinking was controlled by $\mathrm{S}-$ and argues against the possibility that it occurred as a vacuum activity. Control subjects did not confine drinking to the extinction component of the multiple schedule. Instead, they developed a postpellet drinking pattern, which suggests that drinking was controlled by postfood time in the pseudo discrimination. During the generalization test, control rats did not differentially respond to the test stimuli, indicating that changes in responding in the discrimination group were not simply the result of test stimulus novelty. Together, these data provide support for the general account of interim responding proposed by Staddon (1977; Staddon \& Simmelhag, 1971).

Despite the overall form of the interim gradient around S- for Group TDT-, the test stimuli had a comparatively short-term effect on drinking during the generalization test (see Figure 3). This occurred in spite of the presentation of only a very few test trials per session. This flattening of the gradient over test sessions may have been partially due to the testing procedure itself. Because the test stimuli were quite similar to $S-$, and had the same predictive value as $\mathrm{S}-$, the subjects may have learned rapidly that these also predicted the $a b$ sence of food and may have soon begun drinking in the presence of test stimuli as well. Another possibility is that drinking was controlled by more than one stimulus in the present paradigm. Not only did $\mathrm{S}$ - predict the absence of food, but the absence of $S+$ was also a valid signal for this condition and therefore could have controlled drinking. Moreover, as will be discussed below, it seems likely that drinking was partially controlled by postprandial stimuli (e.g., dry mouth, taste and texture of food) and thirst. When tone frequency was varied during the generalization test, the subjects may have attended to more reliable cues, namely thirst and the absence of St, to determine when and how much to drink, thereby making the differential control by the various tone stimuli relatively short-lived.

The acquisition of stimulus control has been traditionally assessed only in terms of change in the rate or probability of some terminal response. For example, with generalization test procedures, excitatory stimulus control is operationally defined by a peaked decremental gradient for terminal responding with a maximum at $\mathrm{S}+$, and inhibitory control by a U-shaped gradient with a minimum at S- (e.g., Terrace, 1966). To a large degree, this manner of measuring control by $\mathrm{S}$ - reflects fundamental assumptions about the nature of inhibitory processes; that is, $\mathrm{S}-$ is assumed to simply reduce the potential for terminal responding, but otherwise has little effect on behavior. An alternative perspective, which views inhibition and excitation as largely parallel processes, has recently gained popularity in the Pavlovian literature (e.g.. Dickenson \& Dearing, 1979; Konorski, 1967; Pearce \& Hall, 1980). According to these accounts, reinforcement and nonreinforcement activate different motivational states. Each state is assumed to have a reciprocal inhibitory connection to the other. During appetitive conditioning, repeated presentation of a stimulus predicting no food results in the formation of an association between the internal representation of the predicting stimulus and a no-food motivational state. In this way, the presentation of S- eventually comes to activate the "no-food" state. As Staddon (1977) has suggested, such a state could provide the motivation for interim behavior. From this perspective, $\mathrm{S}-$ gains not only inhibitory control over terminal behavior, but excitatory control over interim behavior as well. Symmetrically, S+ gains inhibitory control over interim behavior and excitatory control over terminal behavior.

The present study also attempted to assess stimulus control of a putative terminal behavior, contacts with the food magazine. Magazine contacts did not distinguish the true from the pseudo discrimination and were not influenced by test stimuli during the generalization test. For some subjects exposed to the true discrimination, magazine contacts predominated in $\mathrm{S}-$. Observation of these animals suggested that they were attempting to dig through the chamber wall to the source of food. In fact, one rat spent the latter portion of each S- trial chewing through the aluminum wall around the magazine. Staddon (1977) has argued that terminal behavior is related to the acquisition and consumption of food, whereas interim behavior is not food related. But these observations suggest that food-related activities can occur as interim activities. Thus, this aspect of Staddon's theory, and the implications derived from it, may require change.

\section{Role of Postprandial Stimuli}

Although the results of the generalization test indicate that an associative relationship develops between $\mathrm{S}-$ and interim drinking, other data suggest that drinking was also under the partial control of postprandial stimuli. During generalization test trials, the latency to drink appeared to be shorter when more food pellets were presented during the preceding $S+$ trial. Furthermore, data from a single rat during discrimination sessions showed the number of licks (or bout duration) in $\mathrm{S}$ - to be closely related to the amount of food presented in the preceding $S+$ trial. The simplest explana- 
tion for these data seems to be that thirst, and hence drinking, increased with the amount of dry food consumed in St. This interpretation is consistent with the results of other studies showing that the characteristics of the reinforcer which influence thirst (e.g., salt, water, and sugar content) also affect the magnitude of scheduleinduced drinking (e.g., Christian, 1976; Colotla \& Keehn, 1975; Falk, 1967; Poling, Krafft, Chapman, \& Lyon, 1980; Stein, 1964).

As the present data indicate, however, a simple postprandial explanation (e.g., Lotter, Woods, \& Vasselli, 1973; Stein, 1964) is not sufficient to account for interim drinking. If it were, drinking would have followed each food presentation in $S^{+}$rather than occurring in $\mathrm{S}-$, as it did for rats exposed to the true discrimination. Moreover, other studies have shown that postprandial stimuli are not necessary for interim drinking. For example, Cantor and Wilson (1979) observed interim drinking during a schedule of intermittent brain stimulation. Postprandial stimuli are obviously absent in this preparation. Together, these findings suggest that postprandial stimuli are neither necessary nor sufficient for schedule-induced drinking. This raises the question of how these factors might have combined with the predictive properties of $S-$ to produce the pattern of $S_{-}$ drinking seen in this study.

One possibility is that eating dry food acted to select drinking over other possible interim activities by producing some initial motivation to drink. In the multiple schedule, thirst could not have been satisfied in $\mathrm{S}+$, since rats tended to station themselves in front of the food magazine, which was located on the opposite end of the front wall from the water tube. Initially, S- may have provided an opportunity to drink by signaling a food-free period and inhibiting competing terminal behavior. As the present data indicate, drinking eventually came under the associative control of $\mathrm{S}-$; it may have come under its motivational influence as well. In this way, thirst produced by eating dry food would have been enhanced in S-. This interpretation would account for both the location and excessive nature of interim drinking and the relationship between the amount of food consumed in St and water drunk in S-.

\section{Implications for the Study of Interim Behavior}

This type of explanation suggests that interim behavior might be better understood in terms of two processes: selection and induction. Selection refers to the process by which one or more activities come to predominate during the interim period. Induction refers to the exaggeration of these activities beyond their normal level. In their functional analysis of schedule-induced behavior, Staddon and Simmelhag (1971) proposed that interim responding reflects the need to efficiently allocate time to several activities. When an animal is confined in an experimental chamber, it is indeed efficient to satisfy some other need, rather than to be inactive, at times when hunger cannot be satisfied. Staddon (1977) proposed a modified Pavlovian hypothesis to account for the exaggeration of these activities during $\mathrm{S}$ - periods. Presumably, an interim state induced by $S$ - acts as a nonspecific source of motivation for activities other than the terminal response. But relatively little has been said about how one activity typically comes to predominate in the interim period. The notion of a second, selection process addresses this question and seems to complement Staddon and Simmelhag's functional analysis of interim behavior.

Several possible mechanisms could govern the selection of interim behavior, and any of several models of choice could be applied to this problem. As an example, consider one of the simpler models, which suggests that subjects allocate time to activities in proportion to their relative reinforcing value (Baum \& Rachlin, 1969). For the hungry subject, the most valued activity is most likely eating, and therefore that should occupy the greatest proportion of session time. However, during a periodic food schedule, access to food is restricted and is least likely after a food delivery. Because of this temporal constraint, subjects may treat the postpellet period as a choice situation in which eating is not a possible alternative. If this is the case, subjects should select and allocate time to the other alternatives having the greatest relative value. This approach has certain advantages. For example, we should be able to predict which of several possible alternatives will predominate during an interim period by allowing free access to all alternatives and measuring time allocation to each activity (Premack, 1965). The activity or activities receiving the greatest time allocation in the free-choice situation should be selected to fill the interim period when food is periodically presented. Furthermore, any manipulation which changes the value of an activity relative to other alternatives should be reflected in time allocation to that activity. It seems reasonable to assume that the relative value of drinking should increase as thirst increases. Thus, as the salt content of the food reinforcer increases, a greater portion of the interim period should be spent drinking, resulting in greater overall water consumption (Poling et al., 1980). Greater drinking should also occur when water is sweetened with saccharin or sucrose because of its enhanced hedonic quality (e.g., Christian, 1976).

In conclusion, this formulation suggests two lines of research. Several questions need to be addressed concerning how interim responses are selected. For example, Staddon's (1977) analysis implies that only nonfood activities should predominate during an interim period. Alternatively, Rachlin and Buckhard (1978) proposed that interim activities were substitutes for eating. Yet, it is still unclear whether food-related activities can occur as interim responses. Furthermore, it is not clear whether the advent of an interim response can be predicted from its relative reinforcing value as we have outlined here. 
Roper (1978) measured time allocation to several potential interim activities during a free-access procedure and then a periodic food schedule. Interim responding was not predicted in a simple way by time allocation during the free-access procedure. Yet another possibility is that subjects select the activity whose bout duration most closely matches the perceived duration of the interim period (Dunham \& Cornwall, Note 1). Questions concerning the motivation and control of interim behavior have been difficult to address when behavior is maintained by simple periodic schedules, a major problem being that behavior is under temporal control. However, the successive discrimination procedure used in this investigation eliminates many of these difficulties and extends the generality of schedule-induced behavior to another paradigm. Although the present results provide evidence of associative control of drinking by $\mathrm{S}_{-}$-, they do not address the question of motivation. But it should be possible to determine whether interim behavior varies with the strength of an $\mathrm{S}$ - using a modification of the present procedure.

As a final note, it ultimately may be possible to account for all aspects of schedule-induced behavior with a single mechanism. The two-process approach to interim behavior developed here is necessarily less parsimonious than one positing a single process (e.g., Falk, 1971; Staddon, 1977). But this division of theory seems justified by the available literature and is more likely to account for the diversity and excessive nature of the behavior appearing in $\mathrm{S}-$ periods. Moreover, the principal advantage to this formulation is its heuristic value: looking at interim activities from several different perspectives can only lead to a clearer understanding of this class of behavior.

\section{REFERENCE NOTE}

1. Dunham, P. J., \& Cornwall, A. Structural features in multiple response repertoires: Effects of response restrictions. Manuscript in preparation, 1982.

\section{REFERENCES}

Baum, W. M., \& Rachuin, H. C. Choice as time allocation. Journal of the Experimental Analysis of Behavior, 1969, 12, 861-874.

BoAke8, R. A. Performance on learning to associate a stimulus with positive reinforcement. In H. Davis \& M. Hurwitz (Eds.), Operant-Pavlovian Interactions. Hillsdale, N.J: Erlbaum, 1977.

Brown, P. L., \& Jenkins, H. M. Autoshaping on the pigeon's key peck. Journal of the Experimental Analysis of Behavior, $1968,11,1-8$.

Cantor, M. B., \& Wilson, J. F. Polydipsia induced by a schedule of brain stimulation reinforcement. Learning and Motivation, $1979,9,428-445$.

Christian, W. P. Control of schedule-induced polydipsia: Sugar content of the dry food reinforcer. Psychological Record, 1976, 26, $41-47$.

Conen, P. S., \& Looner, T. A. Schedule-induced mirror responding in the pigeon. Journal of the Experimental Analysis of Behavior, 1973, 19, 395-408.
Colotha, V. A., \& Keehn, J. D. Effects of reinforcer-pellet consumption on schedule-induced polydipsia with alcohol, water, and saccharin. Psychological Record, 1975, 25, 91-98.

Dickinson, A., \& Dearina, M. F. Appetitive-aversion interactions and inhibitory processes. In A. Dickenson \& R. A. Boakes (Eds.), Mechanisms of learning and motivation. Hillsdale, N.J: Erlbaum, 203-232.

Fatk, J. L. Control of schedule-induced polydipsia: Type, size, and spacing of meals. Journal of the Experimental Analysis of Behavior, 1967, 10, 199-206.

FaLK, J. L. The nature and determinants of adjunctive behavior. Physiology \& Behavior, 1971, 6, 577-588.

Gamzu, E., \& Schwartz, B. The maintenance of key pecking by stimulus contingent and response-independent food presentations. Journal of the Experimental Analysis of Behavior, 1973, 19, 65-72.

Hutchingon, R. R., Azrin, N. H., \& Hunt, G. M. Attack produced by intermittent reinforcement of a concurrent operant response. Journal of the Experimental Analysis of Behavior, 1968, 11, 489-495.

KILleEn, P. On the temporal control of behavior. Psychological Review, 1975, 82, 89-115.

Konorsxi, J. Integrated activity of the brain. Chicago: University of Chicago Press, 1967.

LEvitskY, D., \& Collier, G. Schedule-induced wheel running. Physiology \& Behavior, 1968, 3, 371-573.

LotTer, E. C., Woons, S. C., \& VAsselli, J. R. Scheduleinduced polydipsia: An artifact. Journal of Comparative and Physiological Psychology, 1973, 83, 478-484.

Pearce, J. M., \& Hall, C. A model for Pavlovian learning: Variations in the effectiveness of conditioned but not unconditioned stimuli. Psychological Review, 1980, 86, 532-552.

Poling, A., Krafft, K., Chapman, L., \& Lyon, D. Polydipsia induced by intermittent delivery of salted liquid food. Journal of the Experimental Analysis of Behavior, 1980, 33, 337-344.

Premack, D. Reinforcement theory. In D. Levine (Ed.), Nebraska Symposium on Motivation: 1965. Lincoln: University of Nebraska Press, 1965.

Rachlin, H., \& BuRxhaRd, B. The temporal triangle: Response substitution in instrumental conditioning. Psychological Review, 1978, 85, 22-47.

REBER, D. Reinforcing the occurrence and nonoccurrence of interim drinking. Animal Learning \& Behavior, 1980, 8, 120-128.

Rebera, D., MANN, B., \& INnis, N. K. Superstitious behavior for food and water in the rat. Physiology \& Behavior, 1977, 19, 803-806.

Rescorla, R. A. Pavlovian conditioning and its proper procedures. Psychological Review, 1967, 74, 71-80.

Resconta, R. A., \& Wagner, A. R. A theory of Pavlovian conditioning: Variations in the effectiveness of reinforcement and nonreinforcement. In A. H. Black W. F. Prokasy (Eds.), Classical conditioning. II. Current research and theory. New York: Appleton-Century-Crofts, 1972.

RoDaen, R. S. The number of non-zero post hoc contrasts from ANOVA and error-rate. British Journal of Mathematical and Statistical Psychology, 1975, 28, 71-78.

ROPER, T. J. Diversity and substitutability of adjunctive activities under fixed-interval schedules of food reinforcement. Journal of the Experimental A nalysis of Behavior, 1978, 30, 83-96.

Schwartz, B., \& Gayzu, E. Pavlovian control of operant behavior. An analysis of autoshaping and its implications for operant behavior. In W. K. Honig \& J. E. R. Staddon (Eds.), Handbook of operant behavior. Englewood Cliffs, N.J: PrenticeHall, 1977.

Staddon, J. E. R. Schedule-induced behaviors. In W. K. Honig \& J. E. R. Staddon (Eds.), Handbook of operant conditioning. New York: Appleton-Century-Crofts, 1977.

Staddon, J. E. R., \& Ayres, S. L. Sequential and temporal properties of behavior induced by a schedule of periodic food delivery. Behaviour, 1975, 54, $26-49$. 
Staddon, J. E. R., \& Simmelhag, V. L. The superstition experiment: A re-examination of its implication for the principles of adaptive behavior. Psychological Review, 1971, 78, 3-43.

STE IN, L. Excessive drinking in the rat: Superstition or thirst? Journal of Comparative and Physiological Psychology, 1964, $58,449-454$.

Terrace, H. S. Stimulus control. In W. K. Honig (Ed.), Operant behavior: Areas of research and application. New York: Appleton-Century-Crofts, 1966.

Tinbergen, N. "Derived" activities: Their causation, biological significance, origin and emancipation during evolution. Quarterly Review of Biology, 1952, 27, 1-32.

\section{NOTE}

1. All statistical tests were based on an alpha level of .05 . All $t$ tests were two-tailed.

(Manuscript received March 2, 1982;

revision accepted for publication August 6, 1982.) 